\title{
Internet of Things (IOT) Based Smart Switch
}

\author{
Sejal Bagde ${ }^{1}$, Pratiksha Ambade ${ }^{1}$, Manasvi Batho ${ }^{1}$, Piyush Duragkar ${ }^{1}$, \\ Prathmesh Dahikar ${ }^{1 *}$, Avinash Ikhar ${ }^{1}$ \\ ${ }^{1}$ Dept. of Electronics and Electronics and Telecommunication Engineering, \\ Dr. Babasaheb Ambedkar Technological University, \\ Lonere, India. \\ ("Email: prathmeshdahikar772@gmail.com)
}

\begin{abstract}
As the years progress, our world is becoming more technologically advanced, and humanity will soon be technologically focused. Henceforth, some fundamental measures should be made by individuals in order to develop the advanced next generation technologies. In this perspective, the proposed research work has developed an Android application with a unit comprising of ESP8266 Wi-Fi module, relay, logic level converter module, capacitive touch sensor module and also a Wi-Fi technology has been used to control the switches.
\end{abstract}

Keywords: smart switch, IoT, Wi-Fi technology, Android application

\section{Introduction}

Internet of Things [IoT] plays a crucial role in this technology driven world. The expansion of the internet of things will also result in new development of everything that is linked to the internet. [1]. Due to the growth in wireless technology, some different connections are introduced; they are GSM, WIFI, ZIGBEE, and Bluetooth. Smart switch systems have also attempted to provide efficient, convenient, suitable and safe way for residents especially aged population to access their homes. The introduction $f$ the concept of security in modern homes will have an impact on the technology advancement [2].

Time plays a crucial role in completing all the tasks assigned to the humans and also it is a human nature to expect the completion of scheduled assignments within the allotted time period. The completion of certain tasks at home without their presence necessitates the 
implementation of automation technologies. This can be accomplished by gaining access to the web/internet from anywhere in the world to control and monitor the home-based devices [3]. In this technological era, everyone has the access to Internet. Apart from communicating with other people over the internet, it is now possible to interface and connect with electrical and electronic equipment over the internet in order to manage, monitor, and operate the devices autonomously. By browsing the specified website page, it is typically adaptable and also it can efficiently operate and monitor various devices remotely. The status of gadgets may also be viewed without difficulty. [4]. The proposed model IOT based automation framework has been implemented along with the automated home temperature, light force detector, fire initiation, LPG gas spillage detection, water level marker, automatic fan speed and also an automatic windows/entryway close/open can also be installed. The development of most intelligent framework is the interest of contemporary culture, which is inextricably linked to innovation. With the help of technology, human lives have become easier and convenient [5]. Combining the recent innovations with the environment and people may also play an important role in the development of a new framework that consistently affects the way of human lives. The world is heading towards innovation, without which no one can function properly and also the recent technological innovation plays an indispensable role in our day-to-day existence and it also brings a sense of comfort into our lives [6].

In the proposed IoT based smart switch control system [SSCS], a smart switch has been created and operated by two techniques. Nowadays, users can access and control their homes remotely from any location and at any time by connecting modern homes to the Internet [7]. Cutback in power usage, price, and capacity of new electronics devices are mainly due to the increase in processing power of newly-designed processors.

Firstly, any device that has been connected to Wi-Fi can switch ON/OFF by staying in different location. Secondly in the absence of Wi-Fi, smart switch can also be handled manually. IoT devices can be used to observe and control the mechanical, electrical and electronic systems, which are used in different places for instance: public and private, industrial, institutional or residential) and industrial automation systems [8]. 


\section{Methodology}

\section{Features of the system:}

In this twenty first century, the swift among traditional analogue conventions to the improvised effortless process to obtain the same outcomes will make the life easier and effortless $[9,10]$. With the introduction of touch sensors into the classic see-saw switches, along with elegant and immersive look, the switches won't only be replacing the traditional clicking switches for touch activation i.e. with just a single touch; the switch gets toggled as ON or OFF. Along with touch activation, cell phones are not only used for communication but also to control the triggering operation and cut short the time/effort spent otherwise. Several attempts have been made to ease and alter the traditional methods and understanding on the ways to trigger a switch. The current circuitry helps in easing the monitoring as well as controlling the status of the application. With this, any application can be toggled, ON and OFF, at a point of time and from any corner of the world, provided that both are virtually connected by meeting all the requirements.

\section{Proposed system working:}

The end user can toggle the switches with the help of their fingertips as the switches are made sensitive to finger touch and also mobile phones can be used for obtaining the same output, instead cell phones can also provide an additional ease for controlling the same rom any part of the world by just operating the application. When a user desires to activate the control switches, an initial verification will be performed, in which the device being used for controlling will be verified to see if it is permitted to do so. Later on, the current status will be determined and the information will be presented via app with the status being toggled with only one tap. The app will also display the switch's current state. Block diagram: 


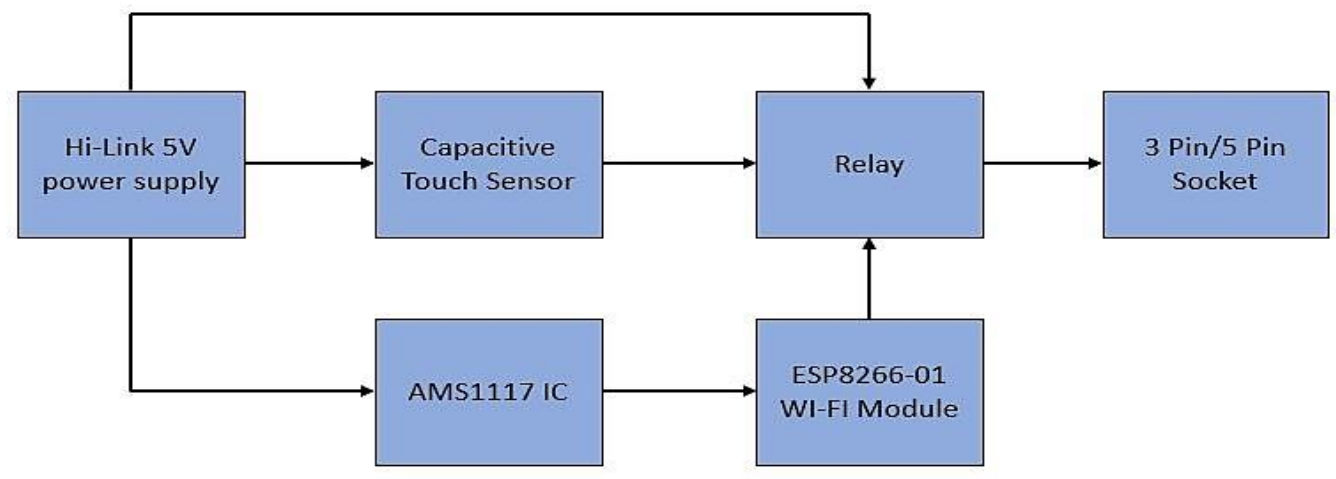

Figure 1. Block diagram of the proposed system

\section{Working Principle}

To operate relay and TTP223 touch sensor, 5v DC power supply is required. So, for power, a Hi-link 5v power supply is utilized along with a function to convert 230v DC to 3v DC. TTP223 is used to control the device and socket in offline mode. The proposed project has replaced the traditional switches with TTP223 touch sensor to give it a modern look. For controlling the device or socket, the config terminal B of TTP223 has been soldered. By doing so, the sensor will be initiated to work. When the user touches the sensor, it sends signal to the relay module in order to turn $\mathrm{ON}$ the device and the similar process will take place, when the sensor is touched again. For controlling the device or socket in online mode, ESP8266-01 Wi-Fi module is utilized. ESP866-01 is controlled by the developed application. The operating voltage of ESP8266-01 is 3.3V. The conversion of voltage AMS1117 H (volatge regulator) is required to convert $5 \mathrm{v}$ DC to $3.3 \mathrm{v}$ DC. The proposed project work has also used some hardware and software tools, they are -

\section{(a)Hardware tools}

\section{ESP8266-01 Wi-Fi Module}




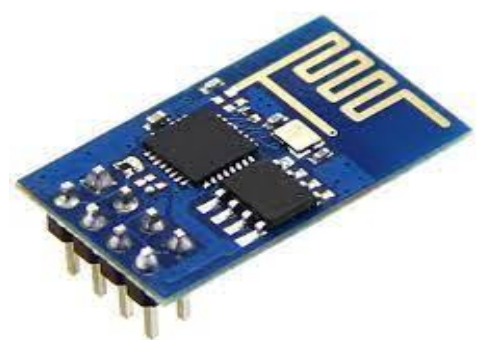

Figure 2. ESP8266-01 Wi-Fi module

ESP8266 is a completely independent Wi-Fi network solution that can control software applications or disable all Wi-Fi networking capabilities via a separate application processor. Despite the fact that the ESP8266 device is ascending and considered as the processor's sole application, the flash memory may be initiated directly from an external source. . Built-in cache memory will help out to improve the system execution and decrease memory demand. Second situation is when wireless internet access presumes the task of WiFi adapter, where it can be attached to any microcontroller-based design; the connection is not difficult, just by SPI / SDIO interface or central processor with AHB bridge interface. Processing and storage capacity on ESP8266 powerful piece can be combined through GPIO ports sensors and other applications specific instruments. The ESP8266 is an extremely nonsegregated chip, which includes anantenna switch, power management converter, least external circuitry, and front-end module by including the whole solution design to reduce the space occupied by PCB. Pins name and functions are given below-

Table 1. Pin configuration of ESP8266-01 Wi-Fi module

\begin{tabular}{|l|l|l|}
\hline $\begin{array}{l}\text { Pin } \\
\text { NO. }\end{array}$ & Pin Name & Function \\
\hline 1. & GND & Ground2 \\
\hline 2. & GPIO2 & $\begin{array}{l}\text { General Purpose input } \\
\text { output; internal pull up }\end{array}$ \\
\hline 3. & GPIO & $\begin{array}{l}\text { General Purpose input } \\
\text { output; internal pull up }\end{array}$ \\
\hline 4. & RXD & $\begin{array}{l}\text { UARTO,Data received } \\
\text { pin }\end{array}$ \\
\hline 5. & VCC & Power Supply \\
\hline 6. & RST & External Reset pin \\
\hline 7. & CH_PD & Chip enabled pin \\
\hline 8. & TXD & UARTO, Data send pin \\
\hline
\end{tabular}


Features of ESP8266 Wi-Fi module:-

○ It is an integrated 32 bit low power Microcontroller unit.

- It has an integrated 10 bit ADC.

○ ESP8266 also has an integrated TCP / IP protocol stack.

○ Contains integrated PLL, regulators and power management units

○ Supports almost all types of antennas

○ Supports $2.4 \mathrm{GHz} \mathrm{WiFi}$, WPA / WPA2

○ Supports STA / AP / STA + AP operating modes

○ Supports Smart Link function for Android and iOS devices

\section{Hi-Link $5 \mathrm{v}$ power supply}

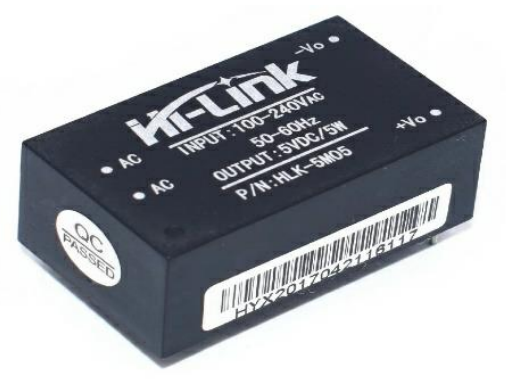

Figure 3. Hi-Link 5v power supply

High link 5V Power Supply module is a plastic encased PCB mounted detached exchanging venture and down power supply module. It can supply $5 \mathrm{~V}$ DC from $120 \mathrm{~V}$ AC $-245 \mathrm{~V}$ AC. This makes it ideal for little ventures, which needs a 5 volt supply from mains. There are numerous benefits for these modules, for example, lowtemperature rise, low force, high productivity, high dependability, high-security confinement and so forth. This power supply module voltage source is an exchanging source, so it does not require any stress over variances in voltage lattice. It is intended to be mounted on the PCB and also it becomes as an ideal answer for the considered power cushions. It is generally utilized in a shrewd home, computerization and control, correspondence hardware, instrumentation and different enterprises. 
Features of Hi-Link power supply:-

- Ultra-thin, ultra-small, the industry's smallest volume

○ Global universal input voltage $(90 \sim 245 \mathrm{Vac})$

○ Low-power, green, no-load loss $<0.1 \mathrm{~W}$

○ Low ripple, low noise

○ Good output short circuit and overcurrent protection and self-recovery.

○ High efficiency and high power density

○ Input and output isolation voltage 3000Vac.

○ $100 \%$ full load aging and testing.

- High reliability, long life design, continuous working time is greater than 100,000 hours.

○ Using high-quality environmentally friendly waterproof plastic potting, moisture, vibration, water and dust to meet IP65 standards.

○ Economic solutions, cost-effective

○ No external circuit to work

III. $\quad 4$ channel Relay module (5v)

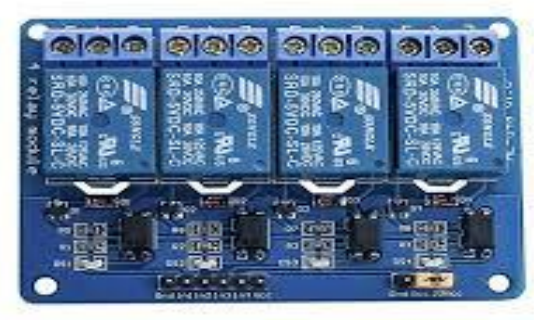

Figure 4.4 channel Relay module (5v)

Relay modules are available for controlling the outside loads. Unlike transistors used for switching, relays use steel transfer contacts, which avoid the strength loss and heating inherent in transistor switching circuits. They include little modules, which are easy to hookup and are specifically beneficial for AC switching. The most important drawback for relays 
is that being an electro-mechanical tool, like all mechanical transfer relays, they are extra vulnerable to put on and tear for an extended use. These relays are completely compatible for $5 \mathrm{~V}$ equipments. Because the inputs feature an integrated relay driving force transistor and opto-isolator, the instrument requires less than five $\mathrm{mA}$ at the excellent judgement control pin to power it. If you've got an Arduino, you may use any of the virtual output pins to power the relays. A good judgement LOW signal is used to activate the relays. The purple LED is switched ON, while the manage pin for that relay is pushed low to set off the relay. When the relay is activated, an audible click on might be heard. A good judgment HIGH deactivates the relay and the LED will move off. The board consists of opto-isolators at the good judgment inputs. Opto-isolators offer whole electric isolation among the good judgment to manage enters and the relay strength as a delivered layer of safety in case of a lighting fixtures strike or a few different type of foremost failure at the AC loads of the relay. As-shipped, the module has a jumper among header pins like JD-VCC and VCC (relay strength is jumper to good judgment strength). This bypasses the opto-isolation circuit since it ties the relay strength to the good judgment strength. Only one strength deliver its wish to be associated to the module and it makes the instrument easier to use. The jumper has been removed to provide a separate $5 \mathrm{~V}$ supply voltage to the JD-VCC and GND pins on that connection if opto-isolation is desired. The module calls for $5 \mathrm{~V}$ strength and GND to operate. If optoisolation is used, it will require specific $5 \mathrm{~V}$ strength sources. The floor has to be in unusual place with the uC. Fly back diodes are covered at the module in parallel with the relay coils to soundly shunt modern-day, while the relay coil is de-energized. When a relay is energized, the module attracts approximately $70 \mathrm{~mA}$ from the Vcc pin. With each relays energized, the draw is ready with $140 \mathrm{~mA}$. The output of the relay is rated to interchange as much as 30VDC at $10 \mathrm{~A}$ according to the relay markings or as much as $125 / 250 \mathrm{VAC}$ at as much as $10 \mathrm{~A}$. The output is SPDT kind with a NO (Normally Open) terminal and a NC (Normally Closed) terminal related to the middle unmarked unusual place (COM) terminal. A good judgment HIGH energizes the relay, so the NO-COM touch is closed and the NC-COM touch is open. A good judgment LOW de-energizes the relay and the NO-COM touch is open and the NCCOM touch is closed. 
Functions of $4 \mathrm{v}$ channel relay module:-

- A relay module has controllable digital output.

○ Relay module is compatible with any $5 \mathrm{~V}$ microcontroller such as Arduino.

$\circ$ Rated through-current: 10A (NO) 5A (NC)

○ Control signal: TTL level

$\circ$ Maximum switching voltage 250VAC/30VDC

- Maximum switching current of a relay module is $10 \mathrm{~A}$

IV. AMS1117 IC

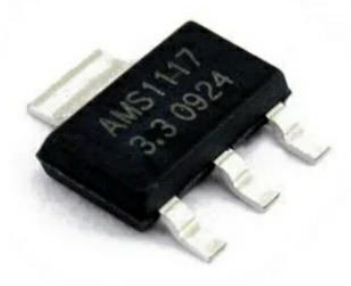

Figure 5. AMS1117 IC

The AMS1117 is a well-known SMD bundle with 3-pin voltage controller that is accessible in numerous models for fixed and flexible voltage prerequisites. The IC can convey a greatest current of $1 \mathrm{~A}$ and the yield voltage can differ from $1.5 \mathrm{~V}$ to $5 \mathrm{~V}$. It additionally includes a low drop out voltage of $1.3 \mathrm{~V}$, while working at the most extreme current. Utilizing the AMS1117 is essentially straight forward. If it's a fixed voltage controller, just power it on, but the Vin pin and the controlled yield may be found on the Vout pin. . The Adj/Ground pin for this situation acts just as a ground pin and it is grounded. Likewise a capacitor can be added at the yield side. For an adjustable kind voltage controller, two outer resistors are required to choose the yield voltage of the regulator, where the resistors R1 and R2 choose the yield voltage of controller. The capacitor CAdj is a discretionary part, which can be added to improve the swell dismissal whenever required. The other two capacitors intend to channel the information and yield clamor individually. 
Features of AMS1117 IC:-

$\bigcirc$ Three Terminal Adjustable or Fixed Voltages 1.5V, 1.8V, 2.5V, 2.85V, 3.3V and $5.0 \mathrm{~V}$

○ Output Current of $1 \mathrm{~A}$

○ Operates Down to $1 \mathrm{~V}$ Dropout

○ Line Regulation: 0.2\% Max.

○ Load Regulation:0.4\% max.

○ SOT-223, TO-252 and SO-8 package available.

- High Efficiency Linear Regulators.

○ $5 \mathrm{~V}$ to $3.3 \mathrm{~V}$ Linear Regulator.

V. Capacitive Touch Sensor Module (TTP223)

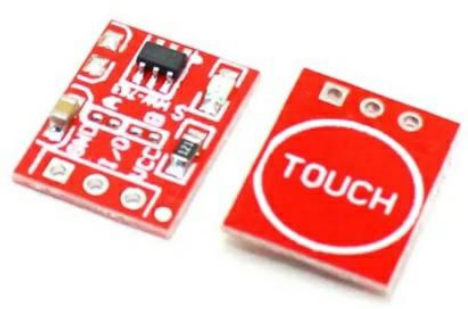

Figure 6. Capacitive Touch Sensor Module (TTP223)

Switch is required to control hardware or electrical machines, sometime electrical switches will give a stun, when electrical switches are used with wet hand and afterward the contact established to control electrical or electronic burden is much intelligent than normal switches, might be a few tasks require contact switch. Here, the Digital Capacitive touch sensor and Arduino interface is made as a test. TTP223B IC based computerized capacitive sensors are truly reasonable and gives great reaction when it has been contacted, this sensor breakout can be effectively interfaced with ESP8266 Wi-Fi module, and it includes just three terminals for outside interface. The TTP223 is a touch cushion indicator IC. which offers 1 touch key. The contacting location IC is intended for supplanting customary direct catch key with assorted cushion size. Capacitive touch permits gadgets to detect when your finger is 
inside a couple of millimeters of a surface to react a catch "press" very much like the functioning of pushbutton. Capacitive detection might be utilized in any place, where TTP223 is 1 Key Touch cushion finder IC, and it is reasonable to distinguish the capacitive component varieties. It devours extremely low force and the working voltage is just between $2.0 \mathrm{~V} \sim 5.5 \mathrm{~V}$

Features of . Capacitive Touch Sensor Module (TTP223):-

$\circ$ Operating voltage $2.0 \mathrm{~V} \sim 5.5 \mathrm{~V}$

○ Operating current @ VDD=3V, no load

○ At low power mode typical $1.5 \mathrm{uA}$, maximum $3.0 \mathrm{uA}$

○ The response time $\max 220 \mathrm{mS}$ at low power mode @ VDD=3V.

- Sensitivity can adjust by the capacitance $(0 \sim 50 \mathrm{pF})$.

○ Stable touching detection of human body for replacing direct switch key.

○ Provide Low Power mode.

○ Provide direct mode toggle mode by pad option(TOG pin).

- All output modes can be selected active high or active low by pad option(AHLB pin)

\section{(b)Software tools}

\section{- MIT App Inventor}

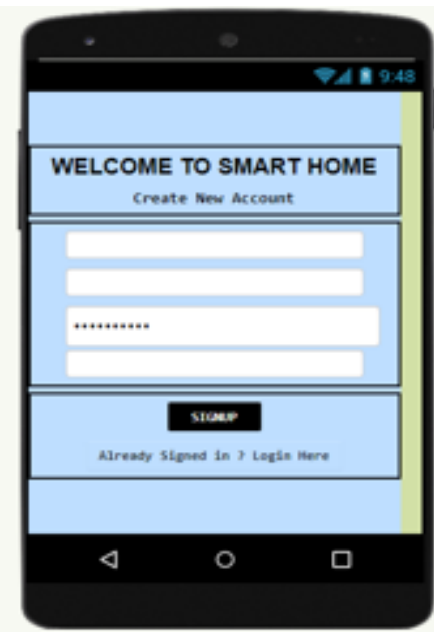

Figure 7. Signup page 
Journal of ISMAC (2021)

Vol.03/ No.02

Pages: 149-162

http://irojournals.com/iroismac/

DOI: https://doi.org/10.36548/jismac.2021.2.007

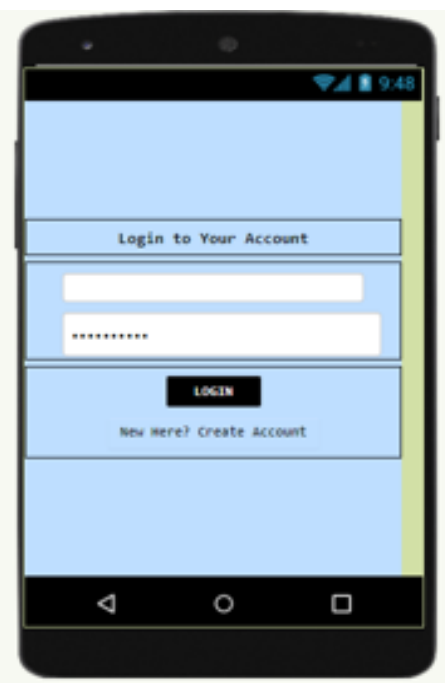

Figure 8. Login page

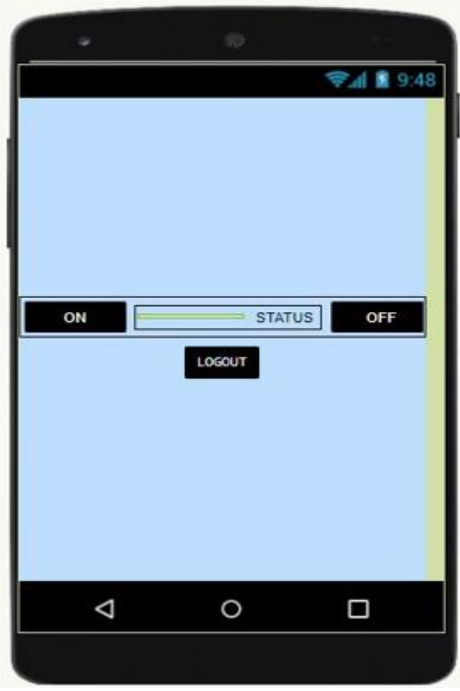

Figure 9. Switch (ON/OFF)

\section{Results and Discussion}

This paper has successfully managed to develop a Wi-Fi-based automation system for smart home and industrial automation using smart switch (Android smart phone). 
Firstly, Android phone gets connected to the nearby Wi-Fi module. The developed applications can be opened in any mobilephone and a username and password should be created before connecting to the device. The username and password can be found in the command prompt. And, now all the electrical appliances can be controlled and it can be introduced to monitor the motion, temperature and humidity of the house.

After the successful connection to the server, the sensor data are sent to the web server for monitoring the system. The web server will allow us to observe and control the system. By entering the assigned user ID and password in server page, the main page will appear. The web server gives the information about the temperature in different places of the room and motion state in the room. It also gives the status of various electrical appliances like light, fan and other appliances, which can be controlled through mobile application or manual switch.

\section{Conclusion}

The proposed research work has designed and constructed an economical Wi-Fi based automation system for smart home and industrial usage prototype by using ESP8266 Wi-Fi module and smart switch (Android smart phone). The smart switch automation system will provide an intelligent and secured system to enhance the quality of human lives. This research work has developed variety of sensors to enhance the safety and security in addition to the fully-automated appliances. The designed system will not only monitor the sensor data like temperature, gas, light, motion sensors, but also it detects the process according to the requirement.

\section{Acknowledgement}

We honestly express our thoughts of gratitude towards our respected guide Prof Avinash Ikhar for his valuable guidance, profound advice, persistent encouragement and his help during the completion of this work. His time to time helpful advice help us to complete this task successfully. Our heartfelt thanks to Head of Department Miss. N. Gyanchandani for providing all kinds of cooperation during the course. Finally, we are thankful to the supporting staff of Instrumentation Engineering Department, JD College of Engineering and management all those who contributed to complete this work. 


\section{References}

[1] Ramlee, Ridza A., Mohd A. Othman, M. H. Leong, Mohd M. Ismail, and S. S. S. Ranjit. "Smart home system using android application." In 2013 International Conference of Information and Communication Technology (ICoICT), pp. 277-280. IEEE, 2013.

[2] Chiu, Min-Chie, Wei-Dian Lai, and Che-Min Chiu. "A smart home system with security and electrical appliances." Journal of Information and Optimization Sciences 42, no. 2 (2021): 303-319.

[3] International Electrotechnical Comission Internet of Things: Wireless Sensor Networks, Geneva, Switherland:International Electrotechnical commission, 2014.

[4] Nicholas D., Darrell B., Somsak S., "Home Automation using Cloud Computing Network and Android/IOS Devices", IEEE Southeastcon 2012, Proceedings of IEEE.

[5] Chan, M., Campo, E., Esteve, D., Fourniols, J.Y., "Smart homes-modern features and upcoming perspectives,” Maturitas, vol. 64, pp. 90-97, 2009.

[6] Hamdan, Yasir Babiker. "Smart Home Environment Future Challenges and Issues-A Survey." Journal of Electronics 3, no. 01 (2021): 239-246.

[7] Jain, Sukrutha A., and Avinash Bharadwaj. "Characterizing WDT subsystem of a Wi-Fi controller in an Automobile based on MIPS32 CPU platform across PVT." Journal of Ubiquitous Computing and Communication Technologies (UCCT) 2, no. 04 (2020): 187-196.

[8] Ranganathan, G. "Real Life Human Movement Realization in Multimodal Group Communication Using Depth Map Information and Machine Learning." Journal of Innovative Image Processing (JIIP) 2, no. 02 (2020): 93-101.

[9] Shrestha, Sujan, and Subarna Shakya. "A Comparative Performance Analysis of FogBased Smart Surveillance System." Journal of trends in Computer Science and Smart technology (TCSST) 202 (2020): 78-88

[10] Shakya, Subarna, and S. Smys. "Reliable Automated Software Testing Through Hybrid Optimization Algorithm." Journal of Ubiquitous Computing and Communication Technologies (UCCT) 2, no. 03 (2020): 126-135. 